\title{
Harmonik bozulma şartlarında transformatör yüklenme kapasitesinin maksimizasyonu için pasif filtre tasarımı
}

\author{
Emine Hümeyra DEMIRCIOĞLU ${ }^{1, *}$, Alp KARADENİZ², \\ Murat Erhan BALCI ${ }^{2,3}$
}

\author{
${ }^{1}$ Uludağ Üniversitesi, Mühendislik Fakültesi, Elektrik-Elektronik Mühendisliği Bölümü, Bursa \\ ${ }^{2}$ Balıkesir Üniversitesi, Mühendislik Fakültesi, Elektrik-Elektronik Mühendisliği Bölümü, Çă̆lş, Balıkesir \\ ${ }^{3}$ Balıkesir Üniversitesi, Yenilenebilir Enerji Araştırma Geliştirme Uygulama ve Araştırma Merkezi, \\ Balıkesir
}

Geliş Tarihi (Received Date): 04.12.2018

Kabul Tarihi (Accepted Date): 02.12.2019

$\ddot{O} \mathbf{z}$

Günümüzde, endüstriyel tesislerin bulunduğu dăğtım sistemlerinde harmonik bozulmaya sahip akım çeken güç elektroniği temelli yükler yaygın olarak kullanılmaktadır. Dolaylsıyla, bu sistemlerde hat akımları ve bara gerilimleri yüksek değerlerde harmonik bozulmaya sahiptir. Harmoniklerin, güç sistem elemanlarında kayıp artışı, güç faktöründe düşüş, dönen elektrik makinalarinda moment dalgalanmalart ile ölçüm ve koruma elemanlarının hatalı çalışması gibi olumsuz etkilere sebep olduğu literatürde bilinmektedir. Bu olumsuz etkileri azaltmak amacıyla harmonik bozulmayı iyileştiren pasif ve aktif filtreler tüketiciler ve enerji servis sağlayıcılar tarafindan yaygın olarak uygulanmaktadır. Pasif filtrelerin tasarıminda geleneksel olarak, her bir harmonik ve toplam harmonik bozulma seviyelerini ve gü̧̧ faktörünü uluslararası standartlarda tavsiye edilen aralıklarda tutmanin yanı sira akım ve/veya gerilim toplam harmonik bozulma indislerinin minimizasyonu, gü̧̈ faktörünün maksimizasyonu, filtre maliyeti ve kayıplarının minimizasyonu amaçlanmış̧ır. Diğer taraftan, literatürdeki yeni çalışmalar, pasif filtreleri, geleneksel harmonik bozulma ve güç faktörü kısıtların dikkate alarak, transformatörlerin harmonik bozulma şartlarinda yüklenme kapasitesini maksimize etmek amacıyla tasarlamıştır. Bu çalışmada, öncelikle, harmonik bozulmaya sahip tipik bir endüstriyel gǚç sistemi için transformatör yüklenme kapasitesinin maksimizasyonunu amaçlayan optimal C tipi pasif filtre tasarım probleminin çözümünde, büyük patlama büyük çöküntü, karınca aslanı optimizasyon ve yusufçuk optimizasyon algoritmaları sonuç ve hiz bakımından karşılaştırmalı olarak analiz edilmiştir. Daha sonra, tasarlanan optimal pasiffiltrenin performansı, kaynağa ait toplam gerilim harmonik bozulmanin, hat

\footnotetext{
* Emine H. DEMIRCIOOĞLU, humeyrametin@ hotmail.com, http://orcid.org/0000-0003-4296-5532

Alp KARADENIZ, akaradeniz@ balikesir.edu.tr, http://orcid.org/0000-0002-0899-6581

Murat Erhan BALCI, mbalci@balikesir.edu.tr, http://orcid.org/0000-0001-8418-8917
} 
empedansı genliğinin ve hat empedansı X/R oranının değişkenlik gösterdiği durumlar için analiz edilmiştir.

Anahtar kelimeler: Harmonikler, pasif filtreler, transformatör yüklenme kapasitesi, optimal tasarim.

\title{
Passive filter design to maximize loading capacity of transformers under harmonically distorted conditions
}

\begin{abstract}
Today, power electronic-based loads, which draw harmonically distorted currents, are widely used in the industrial distribution systems. Thus, line currents and bus voltages have high harmonic distortion levels. It is known in the literature that harmonics cause adverse effects such as loss increase in power system equipment, decrease of power factor, torque pulsations in rotating electric machines, malfunction of measurement and protection devices. Accordingly, to reduce these adverse effects, harmonic mitigation devices as passive and active filters are widely employed by consumers and utilities. For designing passive filters, it is conventionally aimed to achieve different goals as minimization of current and / or voltage total harmonic distortion, power factor maximization and minimization of filter cost and losses while keeping individual / total harmonic distortion levels and power factor in the recommended intervals defined by international standards. On the other hand, recent studies in the literature have designed optimal passive filters to maximize transformer's loading capacity under harmonic distortion conditions while considering conventional optimal passive filter design constraints. In this study, firstly, for the solution of the optimal C-type passive filter design problem, which aims to maximize the loading capacity of the transformer in a typical industrial power system with harmonic distortion, the big bang big crunch, ant lion optimization and dragonfly optimization algorithms was comparatively evaluated in terms of their results and speed. And then, the performance of the designed optimal passive filter was analysed for the cases where the total harmonic distortion of the source, line impedance's magnitude, and line impedance's $X / R$ ratio change.
\end{abstract}

Keywords: Harmonics, passive filters, transformer's loading capacity, optimal design.

\section{Giriş}

Güç elektroniği devreleriyle kontrol edilen yükler ve dağıtık üretim birimleri, modern güç sistemlerinde yaygın olarak kullanılmaktadır. Bu elemanlar, elektrik şebekelerinde hat akımlarının ve bara gerilimlerinin sinüzoidal dalga formundan önemli ölçüde uzaklaşmasına, diğer bir ifadeyle harmonik kirliliğe sahip olmasına yol açmaktadır [1,2]. Harmonik bozulmaya sahip gerilimler ve akımlar, ölçüm ve koruma sistemlerinin hatalı çalışmasına, sistemdeki elemanların kayıplarının artmasına ve aşırı ısınmasına, güç faktöründe düşüşe ve dönen elektrik makinalarında moment dalgalanmalarına sebep olurlar [3, 4]. Bu olumsuz etkileri azaltmak amacıyla harmonik bozulmayı iyileştiren pasif veya aktif filtreler tüketiciler ve enerji servis sağlayıcılar tarafından yaygın olarak uygulanmaktadır [5, 6]. 
Aktif filtreler, hatta seri bağlı gerilim kaynağ olarak kontrol edilebilen güç elektroniği devreleridir. Aktif filtreler, pasif filtrelerle karşılaştırıldığında, harmonik iyileştirme ve reaktif güç kompanzasyonu bakımlarından üstün performansa sahiptirler. Bununla birlikte, özellikle büyük anma güçleri için, yüksek maliyetlidirler [5, 6]. Dolayısıyla, pasif filtreler günümüz güç sistemlerinde halen yaygın olarak tercih edilmektedir [7-9].

Direnç, bobin ve kondansatör elemanlarından oluşan pasif filtreler, şebekeye bağlantı şekillerine göre, seri ve paralel olarak iki sınıfa ayrılırlar [5, 6]. Seri pasif filtreler, kaynak ve yük arasında harmonik akımının akışını engellemek için ayarlandıkları frekansta yüksek empedans gösterirler. Paralel pasif filtreler ise, yük akımı harmoniklerinin toprağa akmasını ve böylece şebeke tarafına geçmesini önleyecek şekilde tasarlanırlar. Bunun için ayarlandıkları harmonik numarasında, düşük empedans gösterirler. Paralel pasif filtreler, harmonik filtrelemenin yanı sıra temel harmonik reaktif güç kompanzasyonu yetenekleri sebebiyle pratikte, seri pasif filtrelerden daha çok kullanılırlar [10]. Diğer taraftan, paralel pasif filtrelerin, tek ayarlı, çift ayarlı, üç ayarlı, sönümlü- çift ayarlı, birinci dereceden yüksek geçiren, ikinci dereceden yüksek geçiren, üçüncü dereceden yüksek geçiren ve C tipi filtre çeşitleri vardır [10-12].

Literatürde, akım toplam harmonik bozulması (THDI), gerilim toplam harmonik bozulması (THDV), güç faktörü (PF), filtre kaybı (FL) ve filtre maliyeti (FC) parametleri dikkate alınarak pasif filtrelerin optimal tasarımları gerçekleştirilmiştir. Buna göre, PF'nin maksimizasyonu ve THDV, THDI, FL ve FC'nin minimizasyonu, optimal pasif filtre tasarım problemlerinde geleneksel olarak hedeflenmiştir [13-15]. Bu çalışmalarda, IEEE 519 standardında [16] tanımlı harmonik sınırları ve istenilen temel frekans güç faktörü aralığı optimizasyon probleminin kısıtları olarak tanımlanmıştır. Ayrıca, yeni tarihli bazı çalışmalarda $[17,18]$ ise güç sistemlerinin IEEE 519 standardında tanımlı harmonik sınırları aşmadan barındırabileceği azami fotovoltaik dağıtık üretim birimi gücünü maksimize etmeyi amaçlayan optimal pasif filtre tasarımları sunulmuştur.

Geleneksel harmonik bozulma ölçüm indisleri olan THDI ve THDV, akım ve gerilim dalga şekli bozulmalarının toplam miktarını gösteren birer indistir. Ancak, bu iki indis akım ve gerilim harmoniklerinin frekanslarını dikkate almazlar [19]. Dolayısıyla, THDI indisi harmonikli akımlar altında transformatör sargılarının frekansa bağlı kayıplarının ve yüklenme kapasitesinin hesabında yetersizdir. Bu eksiği gidermek amacıyla, IEEE standart C57.110-2008 [20], harmonikli akımlar altında transformatörlerin yüklenme kapasitesinin hesabında kullanılmak üzere harmonik kayıp faktörü indisini ( $\left.\mathrm{F}_{\mathrm{HL}}\right)$ tanımlamıştır. Buna göre, [21, 22] çalışmalarında, harmonik bozulmaya sahip kaynak gerilimi olan tipik bir endüstriyel güç sisteminde, doğrusal olmayan yükleri besleyen transformatörlerin yüklenme kapasitesini maksimize etmek için optimal tek-ayarlı ve C tipi pasif filtre tasarımları önerilmiştir. Her iki optimal tasarımın amaç fonksiyonu $\mathrm{F}_{\mathrm{HL}}$ indisinin minimizasyonu olarak seçilmiştir. Bu tasarım çalışmalarında kısıt olarak, optimal pasif filtre tasarım probleminin geleneksel kısıtları dikkate alınmıştır. Bu önerilen tasarımların, THDV ve THDI'yı minimize etmeyi amaçlayan geleneksel tasarımlara göre daha yüksek transformatör yüklenme kapasitesi değerlerini sağladığı, karşılaştırmalı analiz sonuçlarıyla gösterilmiştir. Ayrıca, son zamanlarda yapılan bir diğer çalışmada [11], tek ayarlı, çift ayarlı, üç ayarlı, sönümlü çift ayarlı ve C tipi filtrelerin, transformatörlerin harmonikli sistemlerde yüklenme kapasitesinin iyileştirilmesine katkıları karşılaştırmalı olarak analiz edilmiştir. Bu çalışmada sunulan sonuçlar, transformatör yüklenme kapasitesini iyileştirme performansları bakımından pasif 
filtrelerin en iyiden en kötüye; üç ayarlı, sönümlü çift ayarlı, çift ayarlı, C tipi ve tek ayarlı olarak sıralandığını göstermiştir. Aynı çalışmada, balina optimizayon ve parçacık sürü optimizasyon algoritmaları, filtre tasarım probleminin çözümü için uygulanmıştır. Bu iki algoritmanın sonuçlarının birbiriyle örtüştüğ̈̈, ancak balina optimizasyon algoritmasının parçacık sürü optimizasyon algoritmasına göre daha az iterasyon sayısı ile problemi çözdüğü çıktılarına ulaşılmıştır.

Bu çalışmada, öncelikle, [22]'de çalışılan harmonik bozulmaya sahip tipik bir endüstriyel güç sistemi için transformatör yüklenme kapasitesinin maksimizasyonunu amaçlayan optimal C tipi pasif filtre tasarım probleminin çözümünde, büyük patlama büyük çöküntü [23], karınca aslanı optimizasyon [24] ve yusufçuk optimizasyon [25] algoritmaları, sonuç ve hız bakımından karşılaştırmalı olarak analiz edilmiştir. Daha sonra, tasarlanan optimal pasif filtrenin, kaynağa ait toplam harmonik bozulma, hat empedansi genliği ve hat empedansı $X / R$ oranının değiştiği durumlar için performansı analiz edilmiştir.

\section{Materyal ve yöntem}

Bu çalışmada, sunulan analiz sonuçları $[11,22]$ 'de dikkate alınan tipik endüstriyel güç sistemi için gerçekleştirilen simülasyonlarla elde edilmiştir. $\mathrm{Bu}$ sistemin tek hat diyagramı Şekil 1'de verilmiştir. Şekil 1'den görüldüğü üzere analiz sistemi, dengeli ve harmonik bozulmaya sahip kaynak, üç fazlı dengeli doğrusal ve doğrusal olmayan yükler, ortak bağlantı noktasından (OBN'den) yük barasına enerji taşıyan tüketiciye ait transformatör ve C-tipi pasif filtreden oluşmaktadır.

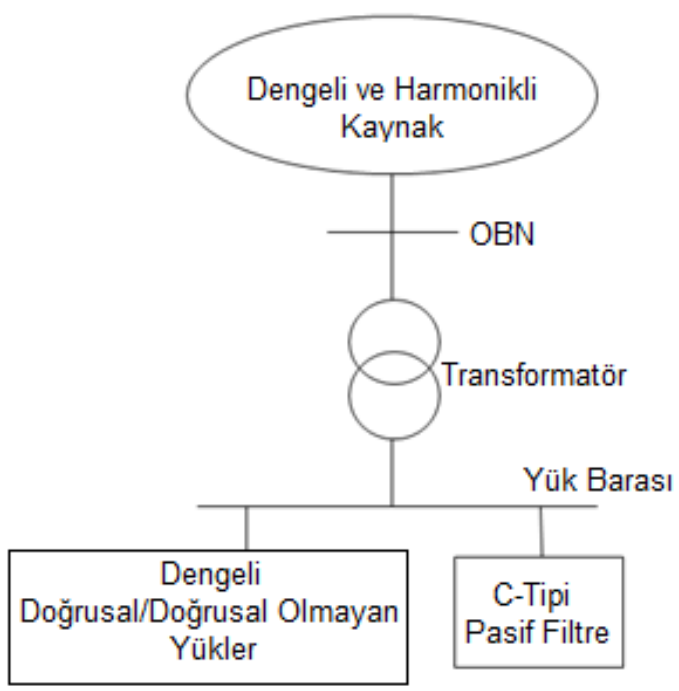

Şekil 1. Tipik bir endüstriyel güç sisteminin tek hat diyagramı.

\subsection{Sistemin modellenmesi}

Şekil 1'de verilen tipik endüstriyel güç sistemininin, tek fazlı eşdeğer devresi Şekil 2'de sunulmuştur. Bu tek faz eşdeğer devre, sistemin dengeli olması sebebiyle akım, gerilim ve güç ifadelerini yazmak için pratik olarak kullanılabilir. Eşdeğer devrede, sistemdeki doğrusal olmayan yükler transformatörün primer tarafından görülen sabit harmonik akım kaynağı $\left(\underline{I}_{L h}^{\prime}\right)$ olarak, doğrusal yükler ise yine transformatörün primer tarafından görülen empedans $\left(R_{L}^{\prime}+j h X_{L}^{\prime}\right)$ olarak modellenmiştir. Sistemde kaynak tarafı, her harmonik 
numarası için bir Thevenin eşdeğer gerilim kaynağı $\left(\underline{V}_{S h}\right)$ ve bir Thevenin eşdeğer empedans1 ( $\left.\underline{Z}_{S h}=R_{S}+j h X_{S}\right)$ olarak modellenmiştir [26-28].

Analiz sisteminde, [29] çalışması göz önüne alınarak, tüketici transformatörü pratik yolla primer taraftan görülen harmonik kısa devre empedansı kullanılarak modellenmiştir:

$\underline{Z}_{T r h}=R_{T r h}+j h X_{T r}$

[30-33] nolu referanslarda sunulan niteliksel ve niceliksel analizlerden, \% 5'in altındaki gerilim toplam harmonik bozulma (THDV) değerleri altında, çekirdek kayıplarının ihmal edilebilir şekilde değiştiği ifade edilebilir. Bu çalışma kapsamında yapılan analizlerde, THDV \%5 değerini aşmadığı için transformatör modelinde çekirdek kayıpları dikkate alınmamıştır.

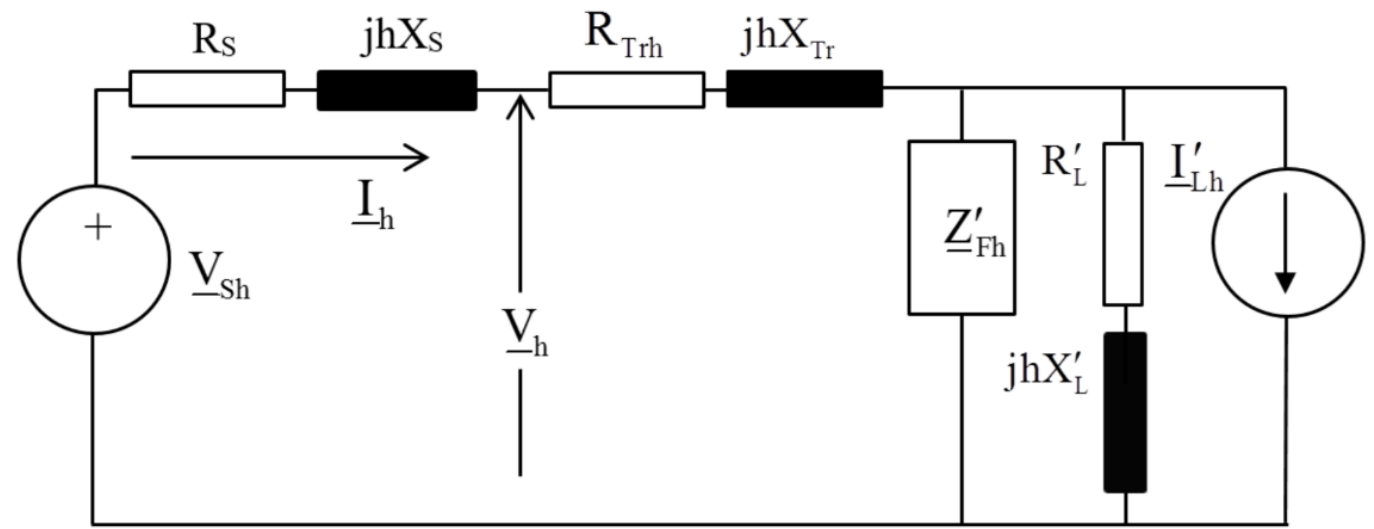

Şekil 2. Tipik bir endüstriyel güç sisteminin tek faz eşdeğer devresi.

Denklem (1)'de, $X_{\operatorname{Tr}}$ sargının temel harmonik endüktif reaktansı ve $R_{\text {Trh }}$ sargının $\mathrm{h}$. harmonik direncini göstermektedir. $R_{\text {Trh }}$ sargının DC direnci $\left(R_{\mathrm{dc}}\right)$ ve sargı kaçak kaybına karşılık gelen direncin $\left(R_{\text {ec }}\right)$ toplamı biçiminde ifade edilebilir [20, 29]:

$$
R_{T r h}=R_{d c}+h^{2} R_{e c}
$$

C tipi filtrenin transformatörün primer tarafından görülen tek faz eşdeğer devresi Şekil 3'de verilmiş olup h. harmonik için empedansı Denklem (3)'de verilen ifadeyle hesaplanabilir:

$$
\underline{Z}_{F h}^{\prime}=-j \frac{X_{C F 1}^{\prime}}{h}+\frac{R_{F}^{\prime} j\left(h X_{L F 1}^{\prime}-X_{C F 2}^{\prime} / h\right)}{R_{F}^{\prime}+j\left(h X_{L F 1}^{\prime}-X_{C F 2}^{\prime} / h\right)}
$$




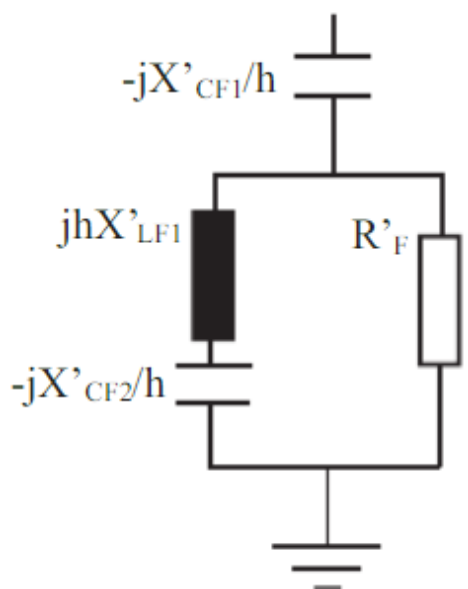

Şekil 3. C tipi filtrenin transformatörün primer tarafından görülen tek faz eşdeğer devresi.

C tipi filtrede bulunan reaktanslar arasındaki ilişkiler Denklem (4)'de verilen şekilde ifade edilebilir:

$$
X_{C F 1}^{\prime}=X_{C F 2}^{\prime}\left(h_{t}^{2}-1\right), X_{L F 1}^{\prime}=X_{C F 2}^{\prime}
$$

Denklem (4)'de $h_{\mathrm{t}}$ filtrenin ayarlandığı harmonik numarasıdır.

\subsection{Güç kalitesi indislerinin hesabı}

Sistemin tek faz eşdeğer devresi dikkate alınarak, Süperpozisyon ilkesine göre; $h$. harmonik için hat akımı $\left(\underline{I}_{h}\right)$ ve OBN gerilimi $\left(\underline{V}_{h}\right)$, Denklem (5) ve (6)'da verilen ifadelerle bulunur:

$\underline{I}_{h}=\frac{\underline{V}_{S h}}{\underline{Z}_{S h}+\underline{Z}_{T r h}+\underline{Z}_{F L h}^{\prime}}+\frac{\underline{Z}_{F L h}^{\prime}}{\underline{Z}_{S h}+\underline{Z}_{T r h}+\underline{Z}_{F L h}^{\prime}} \underline{I}_{L h}^{\prime}$

$\underline{V}_{h}=\underline{V}_{S h}-\underline{I}_{h} \underline{Z}_{S h}$

Burada, $\underline{Z}_{F L h}^{\prime}\left(=\left(\frac{1}{\underline{Z}_{F h}^{\prime}}+\frac{1}{R_{L}^{\prime}+j h X_{L}^{\prime}}\right)^{-1}\right)$, yükün h. harmonik empedansı $\left(R_{L}^{\prime}+j h X_{L}^{\prime}\right)$ ve pasif filtrenin h. harmonik empedansının ( $\underline{Z}_{F h}^{\prime}$ ) paralel eşdeğeridir. Bu ifadelerde, alt simge (_) ilgili gerilim, akım ve empedansların fazör değerlerini belirtir.

Böylece, OBN'deki THDV ve THDI değerleri hesaplanır:

$$
\begin{aligned}
& T H D V=\frac{\sqrt{\sum_{h \geq 2} V_{h}^{2}}}{V_{1}} \cdot 100 \\
& T H D I=\frac{\sqrt{\sum_{h \geq 2} I_{h}^{2}}}{I_{1}} \cdot 100
\end{aligned}
$$


Temel frekans güç faktörü $(D P F)$, temel harmonik aktif gücü $\left(P_{1}=V_{1} I_{1} \operatorname{Cos}\left(\varphi_{1}\right)\right)$ ile temel harmonik görünür gücünün $\left(S_{1}=V_{1} I_{1}\right)$ oranıdır:

$D P F=\frac{P_{1}}{S_{1}}$

IEEE C57.110 standartında tanımlanan $F_{\mathrm{HL}}$ indisinin ifadesi,

$F_{H L}=\frac{\sum_{h} h^{2}\left(\frac{I_{h}}{I_{1}}\right)^{2}}{\sum_{h}\left(\frac{I_{h}}{I_{1}}\right)^{2}}=\frac{\sum_{h} h^{2} I_{h}^{2}}{\sum_{h} I_{h}^{2}}$

olup, sistemde bulunan kuru tip tüketici transformatörünün akım kapasitesi $\left(I_{\text {Maks }}(p u)\right)$ ve yüklenme kapasitesi ( $\left.S_{\text {Maks }}(\%)\right)$,

$$
\begin{aligned}
& I_{\text {Maks }}(p u)=\sqrt{\frac{P_{L L-R}(p u)}{1+F_{H L} P_{E C-R}(p u)}}=\sqrt{\frac{1+P_{E C-R}(p u)}{1+F_{H L} P_{E C-R}(p u)}} \\
& S_{\text {Maks }}(\%)=\sqrt{3} V_{R}(p u) I_{\text {Maks }}(p u) \cdot 100
\end{aligned}
$$

ifadelerine sahiptir.

\subsection{Optimal pasif filtre tasarım probleminin ifade edilmesi}

Literatürdeki $[11,21,22]$ çalışmalarında, transformatörün harmonikli şartlarda yüklenme kapasitesini maksimize etmek için $F_{\mathrm{HL}}$ indisinin minimize edilmesi, optimal pasif filtre tasarım problemi için amaç fonksiyonu olarak seçilmiştir.

OBN gerilimi ve hat akımının her bir harmonik bileşeni ( $V_{h}$ ve $\left.I_{h}\right)$ ve toplam harmonik bozulma değerleri (THDV ve THDI) için IEEE 519 standardında belirtilen sınırlamalar problemin kısıtları olarak tanımlanmıştır. $\mathrm{Bu}$ harmonik kısıtları, ayrıca, rezonans durumuna sebep olabilecek filtre parametrelerinin çözüm olarak bulunmasına engel olacaktır. Filtre bağlandıktan sonra temel frekans reaktif güç kompanzasyonunun istenilen seviyede olması için problemin son kısıtı olarak, $D P F$ 'nin 0.95 ile 1.00 (indüktif) aralığında olması dikkate alınmıştır.

Yukarıda belirtilen amaç ve kısıtlamalara göre, C tipi filtre için optimal tasarım probleminin ifadesi aşağıda verilen biçimde yazılabilir:

Bul: Filtrenin direnç ve reaktans parametreleri (fp),

Amaç Fonksiyonu: Min $F_{\mathrm{HL}}(f p)$

Kisitlar:

$$
\begin{aligned}
& V_{h}(f p) \leq \text { Maks }_{h} \\
& I_{h}(f p) \leq \text { MaksI }_{h}
\end{aligned}
$$




$$
\begin{aligned}
& \text { THDV }(f p) \leq \text { MaksTHDV } \\
& \text { THDI }(f p) \leq \text { MaksTHDI } \\
& 0.95 \leq D P F(f p) \leq 1.00 \text { (indüktif) }
\end{aligned}
$$

Burada, Denklem (13) ve Denklem (14) - (18) sırasıyla problem formülasyonunun amaç fonksiyonu ve kısıtlarıdır. Eşitsizlik kısıtlarında MaksTHDI ve MaksTHDV, IEEE standart 519'da yer alan maksimum izin verilen $T H D I$ ve $T H D V$ değerleridir. $M a k s V_{h}$ ve $M a k s I_{h}$, aynı standartta belirlenen maksimum izin verilen harmonik bileşen değerlerini belirtir.

\subsection{Optimal pasif filtre tasarım probleminin çözüm algoritması}

Bu kısımda, optimal pasif filtre tasarım probleminin çözümüne uygulanan büyük patlama büyük çöküntü, yusufçuk optimizayon ve karınca aslanı optimizasyon algoritmalarının çalışma prensipleri sırasıyla sunulmuştur.

\subsubsection{Büyük patlama büyük çöküntü algoritması}

Büyük patlama büyük çöküntü algoritması, büyük patlama ve büyük çöküntü olarak isimlendirilen iki fazdan oluşmaktadır [23]. İlk fazda çözüm uzayına rasgele bir şekilde çözüm adayları dağıtılır. Bununla birlikte, diğer evrimsel algoritmalara benzer şekilde, başlangıçta (ilk büyük patlama fazında) çözümler, arama alanının her yerine düzgün bir şekilde yayılır. Bu algoritmayı oluştururken, Erol ve Eksin büyük patlamanın rasgele doğasının enerji yayılımıyla ilişkisini dikkate almıştır. Bu durum algoritma perspektifinden bakıldığında, düzenli bir durumdan (yakınsak bir çözümden) kaos durumuna (yeni çözüm adayları grubuna) geçiş manasına gelmektedir.

İkinci faz (büyük çöküntü fazı) ise bir yakınsama operatörüdür. $\mathrm{Bu}$ operatörün birçok girdisi yanında kütle merkezi olarak isimlendirilen tek çıktısı vardır. Kütle merkezi, Denklem (19)'da verilen ifadeyle hesap edilir. Bu denklemde, $\vec{x}^{c}$ kütle merkezini, $\vec{x}^{i}$ nboyutlu uzayda bir çözüm noktası, $f^{i}$ uyum fonksiyonunun $\vec{x}^{i}$ noktasındaki değeri, $N p$ ise büyük patlama fazında oluşturulan popülasyon büyüklüğünü ifade eder.

$\vec{x}^{C}=\frac{\sum_{i=1}^{N p} \frac{1}{f^{i}} \vec{x}^{i}}{\sum_{i=1}^{N p} \frac{1}{f^{i}}}$

Algoritma akışında, bu kütle merkezinin en iyi çözüm olduğu varsayılıp bir sonraki adımda Denklem (20) ve (21) kullanılarak yeni çözümler bu kütle merkezi etrafına yakınsanarak oluşturulur:

$x_{i}^{\text {new }}=X^{c}+\sigma$

$\sigma=\frac{r \alpha\left(x_{\max }-x_{\min }\right)}{k}$

$\mathrm{Bu}$ iki denklemde, $\sigma$ arama uzayını sınırlandırmak için kullanılan parametre, $r$ standart rastgele oluşturulmuş sayı, $x_{\max }$ ve $x_{\min }$ üst ve alt sınır değerleri, $k$ adım sayısını ifade eder. 
Bu patlama ve toplanma işlemleri durdurma kriteri sağlanana kadar devam eder. Denklem (22)'de verilen ifade kullanılarak kütle merkezi ve global en iyi çözüm cinsinden daha iyi yeni çözümler bulunabilir. Bu ifadede $\beta$ global en iyi çözümün kütle merkezine göre öncelik katsayısını, $X^{(k+1, i)}$ yeni adımda üretilen çözüm adaylarını temsil etmektedir.

$X^{(k+1, i)}=\beta X^{c(k)}+(1-\beta) X^{\text {gbest }(k)}+\sigma$

Bu çalışma kapsamında, yapılan denemeler dikkate alınarak, en hızlı şekilde en iyi sonuca ulaşmak için $\beta$ ve $\alpha$ parametreleri sırasıyla 0.2 ve 1 değerlerinde seçilmiştir.

\subsubsection{Karınca aslanı optimizasyon algoritması}

Karınca aslanı optimizasyon algoritması, karınca aslanlarının avlanma tekniğini taklit eden bir optimizasyon algoritmasıdır [24]. Bu avlanma tekniğini kısaca açıklamak gerekirse; karınca aslanı larvaları; bir koni şeklinde dairesel bir yol çizerek karıncalar için tuzaklarını oluştururlar. Bu koloninin gözükmeyen en alt sivri ucuna kendilerini gömerler ve avları olan karıncaları beklemeye başlarlar. Karıncalar gelip, tuzağa girdikleri zamanda tuzaktan çıkmasını engellemek ve tuzağın dibine karıncaları çekmek için, karıncalara kum firlatmaya başlarlar. Yakaladıkları karıncaları ise çeneleri büyük olduğu için hemen yutarlar ve tuzağını tekrar kurup yeni bir karınca gelmesini beklemeye başlarlar.

Buna göre algoritma oluşturulurken, öncelikle karıncaların rasgele yürüyüşlerinin matematiksel modeli Denklem (23)'de verilen biçimde ifade edilmiştir. Bu denklemde, $n$ maksimum iterasyon sayısını, $t$ rasgele yürüyüş adımlarını (iterasyon sayısını), cumsum adımların kümülatif toplam değerini ve $r(\mathrm{t})$ bir rastgelelik fonksiyonunu ifade etmektedir. $r(\mathrm{t})$ rastgelelik fonksiyonu ise Denklem (24)'de verilen biçimde yazılabilir. Bu ifadeye göre, rand'in 0.5 'den küçük eşit olduğu durumlarda $r(\mathrm{t})$ 'nin değeri sifir, rand' in 0.5 'den büyük olduğu durumlarda $r(\mathrm{t})$ 'nin değeri 1'dir.

$$
\begin{gathered}
X(t)=\left(\begin{array}{c}
0 \\
\operatorname{cumsum}\left(2 r\left(t_{1}\right)-1\right) \\
\operatorname{cumsum}\left(2 r\left(t_{2}\right)-1\right) \\
\vdots \\
\operatorname{cumsum}\left(2 r\left(t_{n}\right)-1\right)
\end{array}\right) \\
\mathrm{r}(\mathrm{t})=\left\{\begin{array}{l}
1 \text { if rand }>0.5 \\
0 \text { if rand } \leq 0.5
\end{array}\right.
\end{gathered}
$$

Diğer taraftan, karıncaların rasgele yürüyüşe başladıklarında konumlarını güncellerken arama uzayı sınırlarının dışına çıkılmaması için Denklem (25)'de verilen ifadeye göre konumları normalize edilir. Bu denklemde, $i$ değişken sayısını, $a$ i'nci değişkenin minimum rasgele yürüyüşünü, $b$ i'inci değişkenin maksimum rasgele yürüyüşünü, $c$ ve $d$ ise her bir iterasyonda güncellenen karınca aslanı pozisyonlarının sırasıyla minimum ve maksimum değerlerini ifade etmektedir.

$X_{i}^{t}=\frac{\left(X_{i}^{t}-a_{i}\right)\left(d_{i}-c_{i}^{t}\right)}{d_{i}^{t}-a_{i}}+c_{i}$

Karıncaların tuzağa girdikleri an, karınca aslanının onları tuzağın dibine çekmek için gerçekleştirdiği kum firlatma hareketinin matematiksel modellemesi ise Denklem (26)- 
(29)'da verilmiştir. Bu denklemlerde yer alan, I kaydırma oranını ve Antlion ${ }_{j}^{t} t$. iterasyondaki $j$. karınca aslanını ifade etmektedir.

$c_{i=}^{t}$ Antlion $_{j}^{t}+c^{t}$

$d_{i=}^{t}$ Antlion $_{j}^{t}+d^{t}$

$c^{t}=\frac{c^{t}}{I}$

$d^{t}=\frac{d^{t}}{I}$

Yukarıda verilen denklemlere ilaveten, rulet tekerleği ile seçilen karınca aslanı ve elit karınca aslanı etrafında yürüyen karıncaların yeni pozisyonları Denklem (30)'da verilen ifade kullanılarak bulunabilir. Bu ifadede, $A n t_{i}^{t} t$. iterasyondaki $i$. karınca için; karıncaların yeni pozisyonunu, $R_{A}^{t} t$. iterasyondaki rulet tekerleği tarafından seçilen karınca aslanı çevresindeki rasgele yürüyüşü, $R_{E}^{t}$ ise t. iterasyondaki elit karınca aslanı etrafında rastgele yürüyüşü ifade eder.

$A n t_{i}^{t}=\frac{R_{A}^{t}+R_{E}^{t}}{2}$

Son aşamada ise karınca aslanları tuzağın dibine düşürdükleri karıncaları yedikten sonra kendi pozisyonlarını Denklem (31)'de verilen ifadeye göre güncellerler.

Antlion $_{j}^{t}=$ Ant $_{i}^{t} \quad$ if $\quad f\left(\right.$ Ant $\left._{i}^{t}\right)<f\left(\right.$ Antlion $\left._{j}^{t}\right)$

\subsubsection{Yusufçuk optimizasyon algoritması}

Yusufçuk optimizasyon algoritması, yusufçuk böceklerinin avlanma davranışını taklit eden sezgisel bir optimizasyon algoritmasıdır [25]. Bu algoritmanın çalışma prensibini izah etmek için öncelikle yusufçuk böceklerinin avlanma aşamasını açıklamak faydalı olacaktır. Bu yusufçuk sürülerinin davranışları; Ayırma, Hizalama, Ahenk, Besin ve Düşman öğeleri üzerinden açıklanabilir [34].

Bu öğelerden ilki olan ayırma; sürü içerisindeki diğer yusufçuklarla çarpışmayı önlemek için gereklidir. İkinci öğe olan hizalama; sürü içerisindeki diğer yusufçuklarla hız uyumu için önem arz etmektedir. Diğer öğeler sırasıyla, ahenk; sürü merkezine doğru bireylerin eğilimini, besin; bireylerin gıda kaynaklarına doğru yönelmesini ve düşman ise; sürünün düşmanlardan kaçma eğilimini ifade eder. Algoritma mantığı bakımından ele alındığında, besin optimum çözüme yönelimi, düşmandan kaçış ise tarama alanının artması manalarına gelmektedir.

$\mathrm{Bu}$ algoritma oluşturulurken öncelikle, yusufçuk böceklerinin yukarıda belirtilen davranışları modellenmiştir. Bu modellerden ilki Denklem (32)'de yazılan ayırma ifadesidir. Bu ifadede $S_{i}$; ayırma indisi, $X$ mevcut bireyin pozisyonunu, $X_{j} j$. komşu bireyin pozisyonunu, $N$ ise komşu bireylerin sayısını ifade etmektedir.

$S_{i}=-\sum_{j=1}^{N} X-X_{j}$

Hizalama, ahenk, besin kaynağına çekim ve düşman tehdidi karşısında dışa doğru kaçış davranışlarının modelleri ise sırasıyla Denklem (33)-(36)'da verilmiştir. Bu ifadelerde, 
$A_{i}$; hizalama indisini, $V j$ ise $j$. komşu bireyin hızını, $C_{i}$ ahenk indisini, $F_{i}$; besin kaynağına doğru çekim indisini, $X^{+}$ifadesi besin kaynağının pozisyonunu, $E_{i}$ bir düşman tehdidi karşısında dışa doğru davranış indisini ve $X^{-}$tehdit eden düşmanın pozisyonunu temsil etmektedir.

$\begin{aligned} A_{i} & =\frac{\sum_{j=1}^{N} V_{j}}{N} \\ C_{i} & =\frac{\sum_{j=1}^{N} X_{j}}{N}-X \\ F_{i} & =X^{+}-X \\ E_{i} & =X^{-}+X\end{aligned}$

Yusufçuk böceklerinin $t+1$. iterasyondaki pozisyonları $\left(X_{t+1}\right), t$. iterasyondaki pozisyonlarına $\left(X_{t}\right)$ göre $\Delta X_{t+1}$ adım aralığıyla güncellenir:

$X_{t+1}=X_{t}+\Delta X_{t+1}$

$t+1$. iterasyondaki adım aralığının $\left(\Delta X_{t+1}\right)$ ifadesi, $s$ ayırma, $a$ hizalama, $c$ uyum, $f$ besin, $e$ düşman faktörlerinin ağırlık değerleri ile bu davranışlara ilişkin indisler, $t$. iterasyondaki adım aralığı $\Delta X_{t}$ ve bu adım aralığına ilişkin $w$ atalet ağırlığı cinsinden Denklem (38)'de yazılmıştır:

$\Delta X_{t+1}=\left(s S_{i}+a A_{i}+c C_{i}+f F_{i}+e E_{i}\right)+w \Delta X_{t}$

Ayrıca; bu algoritmada rastgelelilik davranışı ve yapay yusufçukların keşif özelliklerini iyileştirmek için yusufçuk böceklerinin pozisyon denklemi Le'vy uçuşu olarak da tabir edilen rastsal yürüyüş cinsiden ifade edilebilir:

$X_{t+1}=X_{t}+\operatorname{Le}^{\prime} v y(d) * X_{t}$

Bu denklemde, $t$ mevcut döngüyü, $d$ ise pozisyon vektörünün boyutunu temsil etmektedir. Le'vy uçuşunun ifadesi, $r_{1}$ ve $r_{2}$ ile gösterilen iki rasgele say1, $\beta$ sabiti, $\sigma$ fonksiyonu cinsinden Denklem (40)'da verilen biçimde yazılabilir:

$\mathrm{Le}^{\prime} \operatorname{vy}(\mathrm{x})=0.01 * \frac{r_{1} * \sigma}{\left|r_{2}\right|^{\frac{1}{\beta}}}$

Denklem (40)'da yer alan $\sigma$ fonksiyonu ise;

$\sigma=\left(\frac{\Gamma(1+\beta) * \sin \left(\frac{\pi \beta}{2}\right)}{\Gamma\left(\frac{1+\beta}{2}\right) * \beta * 2}\right)^{\frac{1}{\beta}}$

olup burada yazılı Gama fonksiyonunun $(\Gamma(x))$ ifadesi aşağıda verilmiştir:

$\Gamma(x)=(x-1) !$ 


\section{Bulgular ve tartışma}

Benzetimi yapılan sistemin temel frekans kaynak gerilimi ve kısa devre gücü sırasıyla $6345 \mathrm{~V}$ (hat gerilimi) ve 210 MVA'dır. Sistemin tek fazlı eşdeğer devresi için, kaynak empedansı parametreleri ve transformatörün primer tarafından görülen yük empedans parametreleri $\mathrm{R}_{\mathrm{S}}=0.0189 \Omega, \mathrm{X}_{\mathrm{S}}=0.189 \Omega, \mathrm{R}_{\mathrm{L}}{ }^{\prime}=13.85 \Omega$ ve $\mathrm{X}_{\mathrm{L}}{ }^{\prime}=13.18 \Omega \Omega^{\prime} \mathrm{dur}$.

Sistemde, 2 MVA ve $6300 \mathrm{~V} / 400 \mathrm{~V}$ anma değerlerine sahip yıldız-yıldız bağlantılı kuru tip bir tüketici transformatörü bulunmaktadır. Transformatörün $R_{d c}, R_{e c}, X_{T r}$ ve $P_{E C-R}$ parametreleri, $0.104 \Omega, 0.024 \Omega, 0.882 \Omega$ ve 0,231 pu'dur. Bu sistem için kaynak gerilimi ve primer tarafa indirgenmiş yük akımı harmonikleri Tablo 1'de sunulmuştur.

Tablo 1. Kaynak gerilimi harmonikleri ve primer tarafa indirgenmiş yük akımı harmonikleri.

\begin{tabular}{|c|c|c|}
\hline$h$ & $\underline{V}_{S h}(V)$ & $\underline{I}_{L h}^{\prime}(A)$ \\
\hline 5 & $36.64 \angle 0^{\circ}$ & $14.30 \angle 5 \cdot-45^{\circ}$ \\
\hline 7 & $27.48 \angle 0^{\circ}$ & $13.20 \angle 7 \cdot-45^{\circ}$ \\
\hline 11 & $23.82 \angle 0^{\circ}$ & $12.10 \angle 11 \cdot-45^{\circ}$ \\
\hline 13 & $20.15 \angle 0^{\circ}$ & $11.00 \angle 13 \cdot-45^{\circ}$ \\
\hline $17,19,23,25$ & $11.00 \angle 0^{\circ}$ & $7.70 \angle \mathrm{h} \cdot-45^{\circ}$ \\
\hline $29,31,35,37$ & $3.66 \angle 0^{\circ}$ & $5.50 \angle \mathrm{h} \cdot-45^{\circ}$ \\
\hline $41,43,47,49$ & $1.85 \angle 0^{\circ}$ & $3.30 \angle \mathrm{h} \cdot-45^{\circ}$ \\
\hline
\end{tabular}

Pasif filtresiz sistemde, OBN'de ölçülen $T H D V$, THDI ve $F_{H L}$ değerleri \% 3.45, \% 16.19 ve 10.34 'tür. Aynı durumda, $S_{\text {Maks }} \% 60.27$ değerine sahiptir. Yükün $D P F$ ve $P_{1}$ değerleri ise 0.7051 ve $1.410 \mathrm{MW}$ 'dır. IEEE std. 519, analiz edilen sistemin sahip olduğu gerilim seviyesi ve yük akımı/kısa devre akımı oranı için Tablo 2'de verilen gerilim ve akım harmonik sınırlarını önermektedir. Bu tabloda sunulan harmonik sınırlamalar dikkate alındığında OBN'de ölçülen THDV değerinin IEEE std. 519'da belirtilen \%5 sınır değerine uyduğu ancak hat akımının THDI değerinin aynı standartta tanımlı \%15 değerini aştığı görülmektedir. Diğer taraftan $S_{\text {Maks }}$ ve $D P F$ değerlerinin iyileştirilmesi gerektiği ifade edilebilir.

Tablo 2. Analiz sistemi için IEEE std. 519. gerilim ve akım harmonik sınırları.

\begin{tabular}{|l|c|c|c|c|c|l|}
\hline & \multicolumn{5}{|c|}{$\begin{array}{l}\text { Her bir harmonik numaras1 için sinırlamalar } \\
\text { (Temel harmoniğin yüzdesi cinsinden) }\end{array}$} & $\begin{array}{l}\text { THD } \\
\text { Sinır1 }\end{array}$ \\
\hline $\begin{array}{l}\text { Harmonik } \\
\text { numaras1 }\end{array}$ & $h<11$ & $11 \leq \mathrm{h} \leq 17$ & $17 \leq \mathrm{h} \leq 23$ & $23 \leq \mathrm{h} \leq 35$ & $35 \leq \mathrm{h}$ & \\
\hline Hat akım1 & 12 & 5.5 & 5.0 & 2.0 & 1.0 & 15.0 \\
\hline OBN Gerilimi & \multicolumn{7}{|c|}{3.0} & 5.0 \\
\hline
\end{tabular}

\subsection{Optimizasyon algoritmalarının karşılaş̧ırmalı analizi}

Bu kısımda, büyük patlama büyük çöküntü (BPBÇ), karınca aslanı optimizayon (KAO) ve yusufçuk optimizasyon (YO) algoritmalarının dikkate alınan optimal pasif filtre tasarım probleminin çözümünde sonuç ve hız bakımından karşılaştırmalı analizi amaçlanmıştır. Buna göre, BPBÇ, KAO ve YO algoritmaları kullanılarak, optimal pasif filtre tasarım problemi çözülmüş, elde edilen sonuçlar Tablo 3 ve 4'de verilmiştir. 
Tablo 3. BPBÇ, KAO ve YO algoritmaları kullanılarak bulunan optimal C tipi pasif filtre parametre değerleri.

\begin{tabular}{|c|c|c|c|}
\cline { 2 - 4 } \multicolumn{1}{c|}{} & KAO & BPBÇ & YO \\
\hline$X_{C F 1}^{\prime}(\mathbf{\Omega})$ & 27.960 & 27.960 & 27.960 \\
\hline$X_{C F 2}^{\prime}=X_{L F 1}^{\prime}(\boldsymbol{\Omega})$ & 1.090 & 1.093 & 1.090 \\
\hline$R_{F}^{\prime}(\mathbf{\Omega})$ & 6.019 & 5.939 & 5.992 \\
\hline
\end{tabular}

Tablo 4. Tasarlanan filtreler sisteme bağlandıktan sonra ulaşılan güç kalitesi indisleri ve transformatör yüklenme kapasitesi değerleri.

\begin{tabular}{|c|c|c|c|}
\cline { 2 - 4 } \multicolumn{1}{c|}{} & KAO & BPBC & YO \\
\hline THDI(\%) & 12.92 & 12.83 & 12.91 \\
\hline THDV(\%) & 1.74 & 1.74 & 1.74 \\
\hline DPF(\%) & 0.99 & 0.99 & 0.99 \\
\hline $\boldsymbol{F}_{\text {HL }}$ & 1.97 & 1.97 & 1.97 \\
\hline S $_{\text {Maks }}(\%)$ & 91.96 & 91.96 & 91.96 \\
\hline
\end{tabular}

Tablo 3'den, test edilen algoritmalar tarafindan bulunan optimal filtre parametrelerinin $X_{C F 1}^{\prime}=27.960 \Omega, X_{C F 2}^{\prime}=X_{L F 1}^{\prime}=1.09 \Omega$ ve $R_{F}^{\prime}=6.00 \Omega$ civarında değerlerde olduğu görülmektedir. Tablo 4 ise; bu optimal filtre tasarımlarının sağladığı THDI, THDV, $D P F, F_{\mathrm{HL}}$ ve $S_{\text {Maks }}$ değerlerinin yaklaşık olarak, \%12.9, \%1.7, 0.99, 1.97 ve $\% 91.96$ olduğunu göstermektedir. Böylece, test edilen algoritmalar arasında dikkate alınan optimal pasif filtre tasarım probleminin çözümünde ihmal edilebilir farklar olduğu sonucuna varılmıştır.

Tablo 5'den ise, KAO, YO ve BPBÇ algoritmalarının 59, 66 ve 99 iterasyon sayılarında çözüme ulaştığı, dolayısıyla, transformatör yüklenme kapasitesinin maksimizasyonunu amaçlayan optimal pasif filtre tasarım probleminin çözümünde, hız bakımından en iyiden en kötüye algoritmaların, KAO, YO ve BPBÇ olarak sıralandığı görülmektedir.

Tablo 5. Algoritmaların optimal pasif filtre tasarım probleminin çözümü için iterasyon sayıları.

\begin{tabular}{|c|c|}
\hline Algoritma & İterasyon Sayısı \\
\hline KAO & 59 \\
\hline BPBÇ & 99 \\
\hline YO & 66 \\
\hline
\end{tabular}

\subsection{Optimal pasif filtrenin değişken sistem şartlarında performansının analizi}

$\mathrm{Bu}$ kısımda, BPBÇ algoritması kullanılarak elde edilen optimal pasif filtre sisteme bağlıyken; kaynak geriliminin toplam harmonik bozulması $\left(T H D V_{\mathrm{S}}\right)$, hat empedansının $X_{\mathrm{S}} / R_{\mathrm{S}}$ oranı, ve hat empedansının genliğinde $\left(Z_{\mathrm{S}}\right)$ meydana gelen değişimlerin, OBN geriliminin ve hat akımının toplam harmonik bozulma değerlerine (THDV ve THDI), OBN'de ölçülen $F_{\mathrm{HL}}$ değeri ile transformatörün yüklenme kapasitesi ( $S_{\text {Maks }}$ ) üzerine 
etkileri analiz edilecektir. $X_{\mathrm{S}} / R_{\mathrm{S}}$ oranıyla ilgili parametrik analizde $Z_{\mathrm{S}}$ sabit değerde tutulmuş, $Z_{\mathrm{S}}$ ile ilgili parametrik analizde $X_{\mathrm{S}} / R_{\mathrm{S}}$ oranı sabit tutulmuştur.

\subsubsection{Değiş̧en $T H D V_{S}$ değerleri altında elde edilen sonuçlar}

Test sisteminde, IEEE std. 519 dikkate alınarak, $T H D V_{S}$ değeri $\% 0$ ile $\% 5$ aralığında değiştirilmiştir. Bu $T H D V_{\mathrm{S}}$ aralığı için filtresiz sistemde, $F_{\mathrm{HL}}$ ve $S_{\text {Maks }}$ sirasıyla 10.4 ve \%60 etrafında ihmal edilebilir değişimler göstermiştir. Diğer taraftan, optimal pasif filtre tasarımı sisteme bağlıyken, THDV 'nin \%0 ile \%5 aralığı için THDV, THDI, $F_{\mathrm{HL}}$ ve

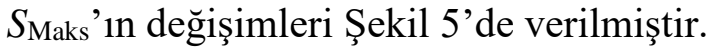

(a)

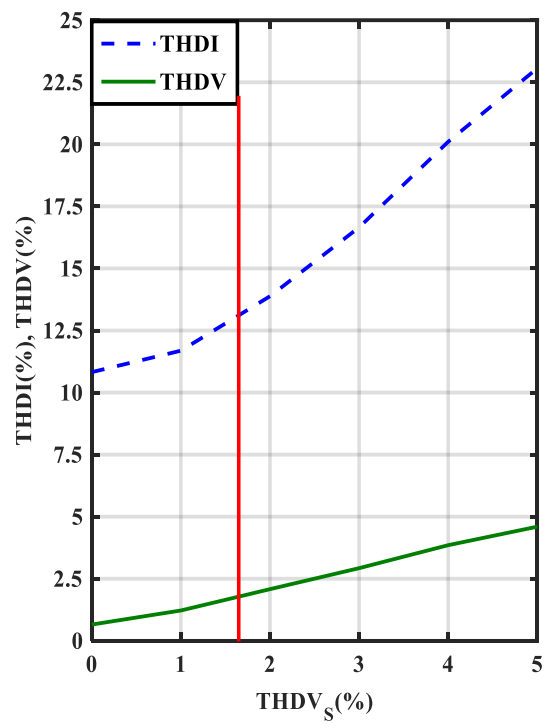

(b)

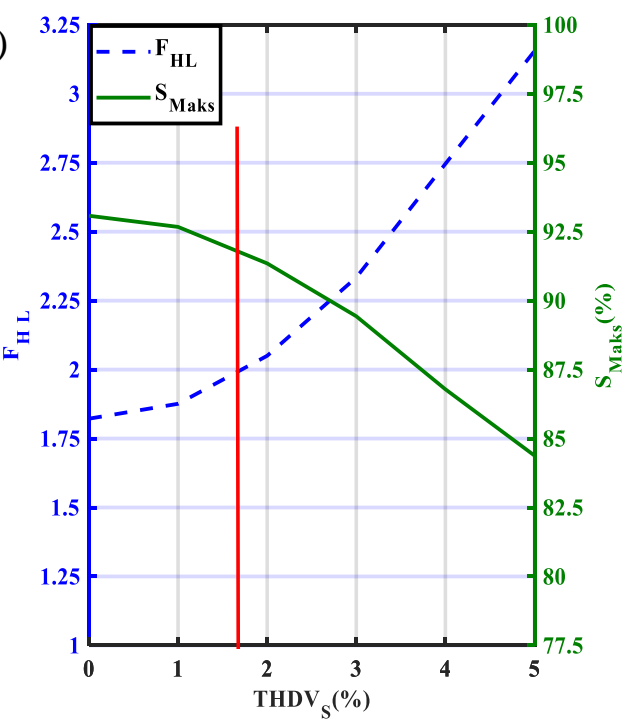

Şekil 5. (a) $T H D V$ ile $T H D I$ indislerinin ve (b) $F_{\mathrm{HL}}$ ile $S_{\text {Maks }}$ indislerinin $T H D V_{\mathrm{S}}$ 'ye bağlı değişimleri.

$\mathrm{Bu}$ şekilde kırmızı çizgi ile işaretli $T H D V_{\mathrm{S}}$ değeri, optimal filtrenin tasarlandığ durumdaki kaynağın toplam harmonik bozulmasıdır. Bu sonuçlara göre, $T H D V_{\mathrm{S}}$ 'nin artışıyla, $T H D V$ 'nin $\% 0$ ile $\% 5$ aralığında, $T H D I$ 'nın $\% 10$ ile $\% 22.5$ aralığında ve $F_{H L}$ 'nin 1.75 ile 3.25 aralığında artış gösterdiği ifade edilebilir. $F_{\mathrm{HL}}$ 'deki bu artışa bağlı olarak $S_{\text {Maks }} \% 92.5$ 'ten $\% 85$ 'e düşmüştür.

\subsubsection{Değişken $X_{S} / R_{S}$ değerleri altında elde edilen sonuçlar}

Analizler sırasında, test sisteminde, hat empedansının $X_{\mathrm{S}} / R_{\mathrm{S}}$ oranı 0.1 ile 100 aralığında değiştirilmiştir. $\mathrm{Bu} X_{\mathrm{S}} / R_{\mathrm{S}}$ aralığ 1 için filtresiz sistemde, $F_{\mathrm{HL}}$ ve $S_{\text {Maks }}$ sırasıyla 10.4 ve $\% 60$ etrafında ihmal edilebilir değişimler göstermiştir. Diğer taraftan, optimal pasif filtre tasarımı sisteme bağlıyken, $X_{\mathrm{S}} / R_{\mathrm{S}}$ 'nin 0.1 ile 100 aralığı için $T H D V, T H D I, F_{\mathrm{HL}}$ ve

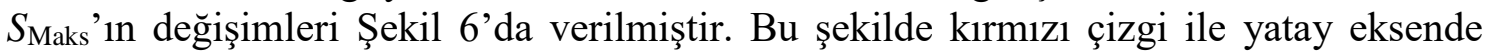
işaretli $X_{S} / R_{S}$ değeri, optimal pasif filtrenin tasarlandığı durumdaki $X_{\mathrm{S}} / R_{\mathrm{S}}$ oranıdır. 
(a)

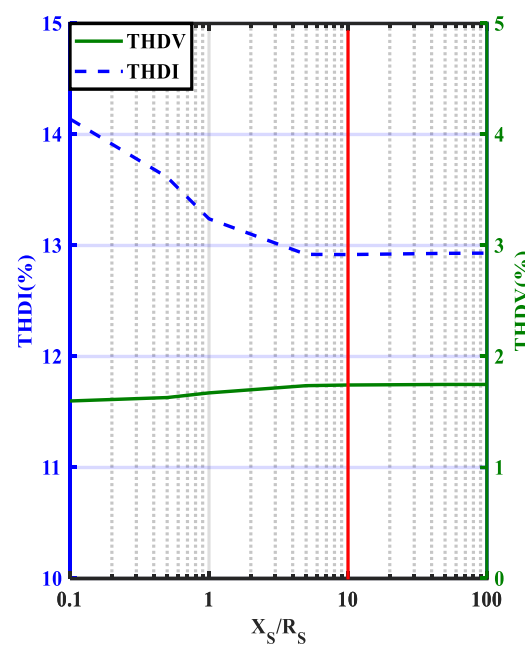

(b)

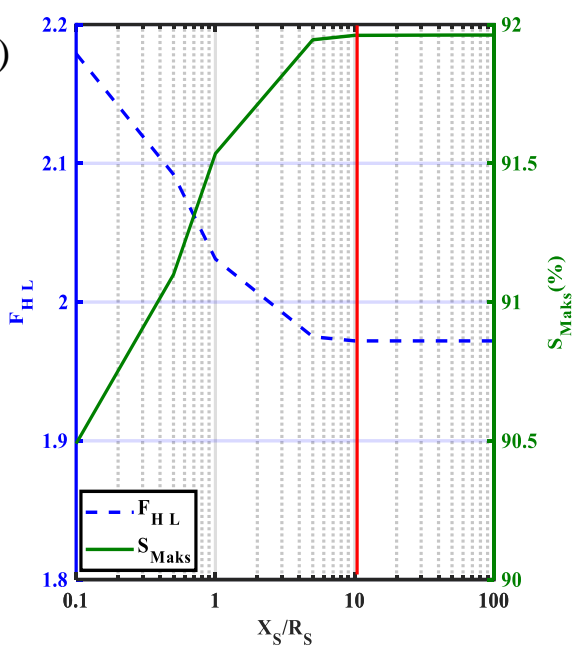

Şekil 6. (a) THDV ile THDI indislerinin ve (b) $F_{H L}$ ile $S_{\text {Maks }}$ indislerinin $X_{S} / R_{S}$ 'ye bağlı değişimleri.

Bu şekilden, $X_{\mathrm{S}} / R_{\mathrm{S}}$ oranının 0.1-10 aralığında artışıyla, THDI'nın \%14'den \%13'e

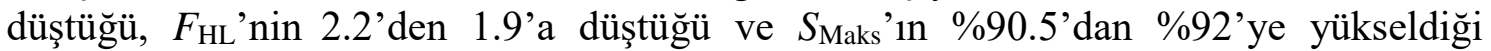
görülmektedir. Bununla birlikte, $X_{\mathrm{S}} / R_{\mathrm{S}}$ oranının 10 ile 100 aralığı için bu üç indiste ihmal edilebilir bir değişim meydana gelmiştir. Ayrıca, analiz sonuçlarına göre tüm $X_{\mathrm{S}} / R_{\mathrm{S}}$ aralığı için $T H D V$ 'nin ihmal edilebilir değişim gösterdiği ifade edilebilir.

\subsubsection{Değişken $Z_{S}$ değgerleri altında elde edilen sonuçlar}

Analizler sırasında, test sisteminde, $Z_{S}$ 'nin normalize edilmiş değeri 0 ile 2.5 aralığında değiştirilmiştir. $Z_{S}$ 'nin normalize değerinin 1 olduğu durum, optimal pasif filtrenin tasarlandığı durumdur. Test edilen $Z_{\mathrm{S}}$ aralığ 1 için filtresiz sistemde, $F_{\mathrm{HL}}$ ve $S_{\text {Maks }}$ sirasiyla 10.4 ve $\% 60$ etrafında ihmal edilebilir değişimler göstermiştir. Optimal pasif filtre tasarımı sisteme bağlıyken, $Z_{\mathrm{S}}$ 'nin 0 ile 2.5 değer aralığı için $T H D V, T H D I, F_{\mathrm{HL}}$ ve

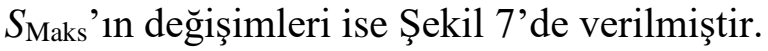

Bu şekilden $Z_{\mathrm{S}}$ 'nin artışıyla, kabaca, THDI'nın \%15'den \%10'a düştüğü, $F_{\mathrm{HL}}$ 'nin 2.2 'den 1.7'ye düştüğü ve $S_{\text {Maks' } 1 n} \% 90$ 'dan \%94'e yükseldiği, THDV'nin ihmal edilebilir miktarda arttı̆̆ 1 ifade edilebilir.

(a)

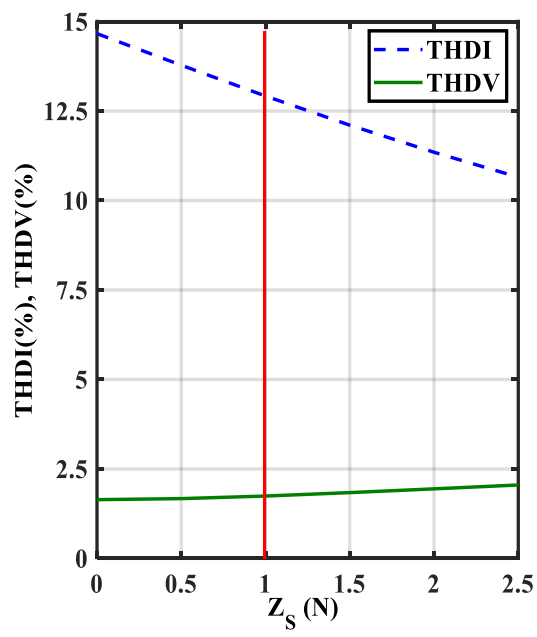

(b)

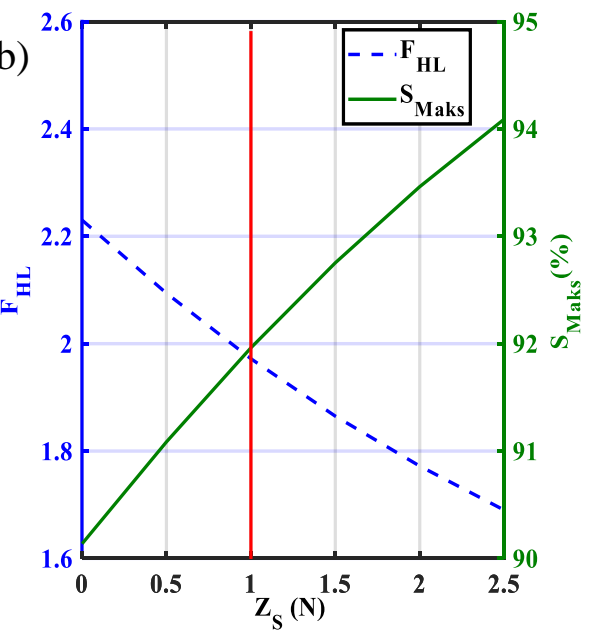

Şekil 7. (a) THDV ile THDI indislerinin ve (b) $F_{H L}$ ile $S_{\text {Maks }}$ indislerinin $Z_{S}$ 'ye bağlı değişimleri. 


\section{Sonuç}

Bu çalışmada, öncelikle, harmonik bozulmaya sahip tipik bir endüstriyel güç sistemi için transformatör yüklenme kapasitesinin maksimizasyonunu amaçlayan optimal pasif filtre tasarım problemi, büyük patlama büyük çöküntü (BPBÇ), karınca aslanı optimizasyon (KAO) ve yusufçuk optimizasyon (YO) algoritmalarıyla çözülmüştür. Elde edilen sonuçlardan, algoritmaların buldukları optimal $C$ tipi pasif filtre parametrelerinin birbirine çok yakın olduğu görülmüştür. Ancak, çözüm iterasyon sayıları karşılaştırıldığında, algoritmaların hız bakımından en iyiden en kötüye, KAO, YO ve BPBÇ olarak sıralandı ğ sonucuna ulaşılmıştır.

Yukarıda belirtilen analizlere ilaveten, optimal C tipi pasiffiltre sisteme bağlı iken kaynak tarafi toplam harmonik bozulmasındaki değişim (THDVS), hat empedansinın $X / R$ oranındaki değişim ve hat empedansının genliğindeki değişimin; transformatörün yüklenme kapasitesi $\left(S_{\text {Maks }}\right)$, harmonik kayıp faktörü $\left(F_{H L}\right)$, ortak bağlantı noktası geriliminin toplam harmonik bozulması (THDV) ve hat akımının toplam harmonik bozulması (THDI) üzerine etkisi analiz edilmiştir. Analizlerden;

- $T H D V_{S}$ artışının, OBN'de ölçülen THDV'yi, hat akımı THDI'sını ve $F_{H L}$ 'yi

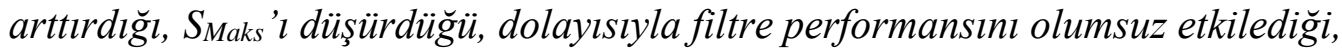

- Hat empedansının X/R oranı ve genliğindeki artışların, OBN'de ölçülen THDV'yi ihmal edilebilir derecede etkilediği, hat akımı THDI'sinı ve $F_{H L}$ 'yi azalttığl, $S_{\text {Maks }}$ l arttırdiğı, böylece filtre performansina olumlu etki ettiği,

sonuçlarına varılmıştır.

\section{Kaynaklar}

[1] Singh, G. K., Power system harmonics research: a survey, European Transactions on Electrical Power, 19, 2, 151 -172, (2007).

[2] Abdul Kadir, A.F., Mohamed, A. ve Shareef, H., Harmonic impact of different distributed generation units on low voltage distribution system, 2011 IEEE International Electric Machines \& Drives Conference (IEMDC), Niagara Falls, 1201-1206, (2011).

[3] Fuchs, E.W. ve Masoum, M.A.S., Power quality in power systems and electrical machines, Elsevier Academic Press, MA, USA, (2008).

[4] Srividhya, J.P., Sivakumar, D. ve Shanmathi, T., A review on causes, effects and detection techniques of harmonics in the power system, IEEE International Conference on Computation of Power, Energy Information and Commuincation, 680-686, Chennai, India, (2016).

[5] Peng, F.Z., Harmonic sources and filtering approaches, IEEE Transactions on Industry Applications Magazine, 7, 4, 18-25, (2001).

[6] Motta, L. ve Faúndes, N., Active / passive harmonic filters: applications, challenges \& trends, 17th International Conference on Harmonics and Quality of Power (ICHQP), Belo Horizonte, 657-662, (2016).

[7] Badrzadeh, B., Smith, K. S. ve Wilson, R. C., Designing passive harmonic filters for an aluminum smelting plant, IEEE Transactions on Industry Applications, 47, 2, 973-983, (2011).

[8] Badrzadeh, B. ve Gupta, M., Practical experiences and mitigation methods of harmonics in wind power plants, IEEE Transactions on Industry Applications, 49, 5, 2279-2289, (2013). 
[9] Dekka, A., Beig, A.R., Kanukollu, S. ve Al Rahis, M.S., Retrofitting of harmonic power filters in onshore oil drilling rigs: challenges and solutions, IEEE Transactions on Industry Applications, 50, 1, 142-154, (2014).

[10] Das, J. C., Power system harmonics and passive filter designs, IEEE Press, (2015).

[11] Karadeniz, A. ve Balci, M.E., Comparative evaluation of common passive filter types regarding maximization of transformer's loading capability under nonsinusoidal conditions, Electric Power Systems Research, 158, 324-334, (2018).

[12] Abdel Aleem, S. H. E., Zobaa, A. F. ve Balci, M. E., Optimal resonance-free thirdorder high-pass filters based on minimization of the total cost of the filters using Crow Search Algorithm, Electric Power Systems Research, 151, 381-394, (2017).

[13] Zeineldin, H.H. ve Zobaa, A.F., Particle swarm optimization of passive filters for industrial plants in distribution networks, Electric Power Components and Systems, 39, 16, 1795-1808, (2011).

[14] Balci, M.E. ve Karacaoglan, A.D., Optimal design of c-type passive filters based on response surface methodology for typical industrial power systems, Electric Power Systems Research, 41, 7, 653-668, (2013).

[15] Churio-Barboza, J.C. ve Maza-Ortega, J.M., Comprehensive design methodology of tuned passive filters based on a probabilistic approach, IET Generation, Transmission \& Distribution, 8, 1, 170-177, (2014).

[16] IEEE 519, IEEE recommended practices and requirements for harmonic control in electrical power systems, IEEE Standardı, (2014).

[17] Sakar, S., Balci, M. E., Abdel Aleem, S. H. E. ve Zobaa, A. F., Increasing pv hosting capacity in distorted distribution systems using passive harmonic filtering, Electric Power Systems Research, 148, 74-86, (2017).

[18] Sakar, S., Balci, M. E., Abdel Aleem, S. H. E. ve Zobaa, Integration of large- scale pv plants in non-sinusoidal environments: considerations on hosting capacity and harmonic distortion limits, Renewable and Sustainable Energy Reviews, 82, 1, 176-186, (2018).

[19] Heydt, C.T. ve Jewell, W.T., Pitfalls of electric power quality indices, IEEE Transactions on Power Delivery, 13, 2, 570-578, (1998).

[20] ANSI/IEEE C.57.110-2008, IEEE recommended practice for establishing transformer capability when supplying nonsinusoidal load currents, IEEE Standardı, (2008).

[21] Balci, M.E. ve Sakar, S., Optimal design of single-tuned passive filters to minimize harmonic loss factor, Middle-East Journal of Scientific Research, 21, 11, 2149-2155, (2014).

[22] Balci, M.E., Optimal c-type filter design to maximize transformer's loading capability under non-sinusoidal conditions, Electric Power Components and Systems, 42, 14, 1565-1575, (2014).

[23] Erol O.K. ve Eksin I., A new optimization method: big bang-big crunch, Advances in Engineering Software, 37, 2, 106-111, (2006).

[24] Mirjalili, S., The ant lion optimizer, Advances in Engineering Software, 83, 8098, (2015).

[25] Mirjalili, S., Dragonfly algorithm: a new meta-heuristic optimization technique for solving single-objective, discrete, and multi-objective problems, Neural Computing and Applications., 27, 4, 1053-1073, (2016).

[26] IEEE Task Force Harmonic Modeling and Simulation, Modeling and simulation of the propagation of harmonics in electric power networks part I: concepts, 
models and simulation techniques, IEEE Transactions on Power Delivery, 11, 1, 452-465, (1996).

[27] McGranaghan, M.F., Dugan, R.C., King, J.A. ve Jewell, W.T., Distribution feeder harmonic study methodology, IEEE Transactions on Power Apparatus Systems, PAS-103, 12, 3663-3671, (1984).

[28] Xu, W., Component modeling issues for power quality assessment, IEEE Power Engineering Review, 21, 11, 12-17, (2001).

[29] Arslan, E., Balci, M.E. ve Hocaoglu M.H., An analysis into the effect of voltage harmonics on the maximum loading capability of transformers, 16th Int. Conf. on Harmonics and Quality of Power, 616-620, Bucharest, Romania, (2014).

[30] Bishop M. T., Baranowski J. F., Heath D. ve Benna S. J., Evaluating harmonicinduced transformer heating, IEEE Transactions on Power Delivery, 10, 1, 305 -310, (1996).

[31] Elmoudi A., Lehtonen M. ve Nordman H., Effect of harmonics on transformers loss of life, Proc. of IEEE Int. Symp. Elect. Insul. Conf. Rec., 408 -411, (2006).

[32] Shareghi M., Phung B. T., Naderi M. S., Blackburn T. R. ve Ambikairajah E., Effects of current and voltage harmonics on distribution transformer losses, International Conf. on Condition Monitoring and Diagnosis (CMD) 2012, 633-636, (2012).

[33] Soh T. L. G., Said D. M., Ahmad N., Nor K. M. ve Salim F., Experimental study on the impact of harmonics on transformer, IEEE 7th Int. Power Eng. and Optimization Conf. (PEOCO), 686-690, (2013).

[34] Katırcıoğlu, F., Renkli görüntüler için yusufçuk algoritması kullanılarak benzerlik görüntüsüne dayalı eşikleme, Düzce Üniversitesi Bilim ve Teknoloji Dergisi, 5, 506-523, (2017). 